\title{
Rediseño del Programa de Estudios de Estadística para la Ingeniería en Alimentos
}

\author{
Redesign of the Statistics for Food Engineering Curriculum
}

Ana Isabel Garcia-Monroy ${ }^{1}$,Engelbert Eduardo Linares-Gonzalez ${ }^{2}$ y Lucero Martinez-Allende 3

\section{(0) \\ EDICIÓN: 曲:CIVTAC}

Recibido: 21/julio/2020

Aceptado: 6/septiembre/2020

Publicado: 25/septiembre/2020

我 México

${ }^{2}$ México

${ }^{3}$ México

\section{IIIIInstitución}

${ }^{1}$ Instituto Politécnico Nacional ${ }^{2}$ Instituto Politécnico Nacional ${ }^{3}$ Instituto Politécnico Nacional

\section{Correo Eletrónico}

1allendeluz@gmail.com

ªgarciamo@ipn.mx

3elinaresg@ipn.mx

\section{(1) ORCID}

${ }^{1}$ https://orcid.org/0000-0003-3646-0386 ${ }^{2} \mathrm{https} / / /$ orcid.org/0000-0003-4814-6562 ${ }^{3}$ https://orcid.org/0000-0002-2924-0118

\section{Citar así: GAAPA / IEEE}

Martinez-Allende, L., Garcia-Monroy, A. \& Linares-Gonzalez, E. (2020). Rediseño del Programa de Estudios de Estadística para la Ingeniería en Alimentos. Revista Tecnológica-Educativa Docentes 2.0, 9(2), 223-230.

https://doi.org/10.37843/rted.v9i2.167

L. Martinez-Allende, A. Garcia-Monroy y E. Linares-Gonzalez, "Rediseño del Programa de Estudios de Estadística para la Ingeniería en Alimentos", RTED, vol. 9, n. ${ }^{\circ}$ 2, pp. 223-230, sep. 2020.

\section{Resumen}

En este proyecto se presentó una propuesta del programa de estudios de la asignatura de estadística, para el rediseño de planes y programas de la carrera de ingeniería en Alimentos en la Unidad Profesional Interdisciplinaria de Biotecnología. Se tomó un grupo piloto de 80 estudiantes, que cursaron esta materia en el segundo periodo del 2018, obteniendo un aumento favorable en su aprovechamiento escolar y una vinculación con otras áreas que se encuentran en su mapa curricular. Al manejar disciplinas interdisciplinares como programación, microbiología, métodos cuantitativos, envases, embalaje, diseño de experimentos, se ha logrado aplicar contenidos esenciales de cada una de ellas al trabajarlas con un conjunto de datos recopilados de un problema planteado, se desarrollan diferentes talentos laborales acorde con manejo de información, se alcanzó un aprendizaje metacognitivo, pensamiento crítico, un manejó en la solución de problemas, creatividad y novedosas creaciones se desarrolló que los llevan a conclusiones verdaderas, cómo el reforzamiento de habilidades en comunicación, colaboración, trabajo en equipo, el estudiante, adquirió nuevas estrategias, al ser capaz de vincular varias materias al mismo tiempo en crear un esbozo experimental.

Palabras clave: Programas de estudios, rediseñ, competencias, habilidades, aprendizaje.

\section{Abstract}

In this project, a proposal for the study program of the statistics subject was presented, for the redesign of plans and programs of the Food engineering career in the Interdisciplinary Professional Unit of Biotechnology. A pilot group of 80 students was taken, who studied this subject in the second period of 2018, obtaining a favorable increase in their school achievement and a link with other areas that are in their curricular map. When handling interdisciplinary disciplines such as programming, microbiology, quantitative methods, packaging, packaging, design of experiments, it has been possible to apply essential contents of each of them when working with a set of data collected from a problem posed, different work talents are developed according to With information management, metacognitive learning, critical thinking, a problem solving management, creativity and innovative creations was developed that lead them to true conclusions, how the reinforcement of communication skills, collaboration, teamwork was achieved., the student, acquired new strategies, being able to link several subjects at the same time in creating an experimental sketch.

Keywords: Curriculum, redesign, skills, skills, learning. 


\section{Introducción}

Ante los informes publicados sobre el manejo de las competencias y habilidades impulsadas por la UNESCO (1998); y OCDE y otras organizaciones mundiales, y el papel que juegan las instituciones educativas de nivel superior, es transcendental realizar modificaciones a los programas de estudio de las diferentes carreras de ingeniería, estos cambios deben propiciar el fortalecimiento y crecimiento de diversos talentos que los lleven a tener éxito laboral y personal.

Las competencias y habilidades no son términos nuevos, la forma como abordarlos es lo novedoso, al modificar los programas de estudio, estos cambios provocan un impacto significativo en los estudiantes, les modifica la visión, que tienen de la profesión que eligieron, si es lo que ellos esperaban, al explorar las opciones de desarrollo profesional y en su vida, hacen que se desarrolle la innovación y creatividad en diferentes áreas de la ingeniería.

Derivado de esto en la unidad profesional interdisciplinaria de biotecnología, se realizó un análisis del programa curricular de la carrera de ingeniería Alimentos, con un estudio sectorial, se encontraron diversas necesidades que requieren los sectores industriales del ramo, acompañado de una encuesta realizada a egresados, cuyos resultados derivaron competencias y habilidades necesarias para lograr tener un éxito laboral.

Del estudio realizado se derivaron diferentes competencias y habilidades requeridas, entre ellas encontramos, la innovación de nuevos artículos, administración de dispositivos de laboratorio, técnicas de fabricación productos como servicios, solución de problemas de operación, toma de decisión, gestión de calidad y certificación, capacidad de coordinar equipos de trabajo, manejo de nuevas tecnologías de la comunicación, dirección de grupos de colaboración, creatividad en el impulso de proyectos, creación de procesos de conservación, diseño de metodologías que aumenten de la productividad, poseer una actitud emprendedora, conocimiento de la legislación vigente, comprensión de las operaciones de los métodos de elaboración de bienes, manejó de personal, aptitud de liderazgo, disposición de trabajar en forma (colaborativo y en equipo).

Cuando los programas de estudios llevan una vinculación entre la universidad y el sector industrial, la interdisciplinariedad, flexibilidad en los aprendizajes, alcanzado tener contenidos curriculares con nuevas competencias y habilidades pertinentes en la creación de metodologías de enseñanza y capacitación. Mostrando lo que realmente necesita el estudiante.

Determina Blázquez (2019), que se requieren competencias con multifuncionalidad, compromiso, servicio, trabajo en equipo, inteligencia emocional. Diversos investigadores en pedagogía han señalado la importancia de integrar, ámbitos temáticos interdisciplinarios, en las áreas básicas, la UNESCO (1998) estableció cuatro categorías de instrucciones para la vida, llamados pilares de la educación en las formas de aprender (hacer, convivir, conocer y ser).

Señala Carneiro (2007) que los alumnos necesitan tener conocimientos académicos, estableciendo un vínculo entre el dominio y la habilidad en la forma de como aprenden (pág. 156). Donde la Cooperación Económica de Asia y el Pacífico, definen a las competencias como conocimientos, talentos y actitudes necesarias, en la participación en una sociedad diversa, y una industria que cambia a gran velocidad.

En el Marco para el aprendizaje del siglo XXI, estableció que las capacidades y habilidades fundamentales que se requieren en el trabajo deben ser impartidas de manera fundamental en la formación y desarrollo profesional, como las competencias sociales e intercalares, descritas en las "4 C" (comunicación, colaboración, idoneidad del pensamiento crítico y creativo) (P21, 2007a, 2013).

Robinson (2006) argumento que el triunfo de las personas depende del modo innovador en que se reestructuren los sistemas educativos. Gardner (2008) \& Sternberg (2007), mencionaron la importancia de la capacidad de "abrir nuevos caminos", en las diferentes maneras de pensar, al proponer ideas, nuevas soluciones, plantear cuestiones desconocidas y llegar a respuestas inesperadas contribuyen a impulsar la imaginación e innovación.

Menciono Barry (2012) algunas competencias que los alumnos necesitan lograr en la inserción exitosa en el trabajo, enunciando al pensamiento crítico, comunicación, liderazgo, colaboración, adaptabilidad, productividad, rendición de cuentas, innovación, ciudadanía mundial, emprendimiento, capacidad para acceder a la información, analizarla y sintetizarla, como talentos fundamentales. 
Plantearon Sternberg \& Subotnik (2006) un plan de estudios centrado en fomentar los talentos de los alumnos en "las 3 R", razonamiento (pensamiento analítico y crítico y capacidades de resolución de problemas), resiliencia (competencias para la vida, como la flexibilidad, la adaptabilidad y la autonomía) y responsabilidad (sabiduría o la aplicación de la inteligencia, la creatividad y el conocimiento en pro de un bien común) (pág. 1).

Mencionaron McLoughlin y Lee (2008) que mientras los alumnos desarrollen un pensamiento creativo alcanzan a realizar un análisis, síntesis, una exploración de diversas fuentes confiables, sé adquirió capacidades en evaluar con objetividad, fiabilidad y actualidad la información, con una comunicación asertiva, al momento se desarrolla una habilidad para examinar, analizar, interpretar datos empíricos.

La capacidad de resolver problemas incluye aspectos $\mathrm{y}$ habilidades que requieren (buscar, seleccionar, evaluar, organizar, sopesar alternativas e interpretar información). Al demostrar las soluciones posibles de diversas dificultades que hoy en día se requiere de una amplia gama de competencias relacionadas con el pensamiento crítico, la innovación y la creatividad (P21, 2007a).

Al señalar Facer (2009) que, al realizar funciones de colaboración y trabajo en equipo, los alumnos van aprendiendo a laborar juntos de manera colaborativa, fortaleciendo sus capacidades, al iniciar esta labor desde los centros educativos, en los ambientes profesionales, se espera que establezcan relaciones que contribuyan a fortalecer sus labores con sus compañeros.

En un discurso pronunciado en 'TED' de 2006, el conferencista Ken Robinson, abordo el tema de la creatividad, afirmó que "nos convertimos en creadores al crecer, qué nuestro crecimiento $\mathrm{y}$ educación proceden de la creatividad". Ante una industria cada vez más automatizada en diferentes áreas, es necesario alentar un espíritu innovador y creativo, cualidades que se solicitan para alcanzar un éxito.

En el cuadro1, se observa diferentes capacidades que requieren los estudiantes hoy en día, se advierte que las actitudes que son demandadas en el 2018 son las mismas para el 2022 estimándose que estos talentos tengan un crecimiento exponencial, mientras que distintas facultades como es las destrezas manuales, habilidades visuales, gestión de recursos financieros, materiales, entre otras exista un decrecimiento.

\section{Cuadro 1}

The Future of Jobs Report 2018.

\begin{tabular}{|c|c|c|}
\hline $\begin{array}{l}\text { Capacidades } \\
\text { demandadas } \\
(\mathbf{2 0 1 8 )}\end{array}$ & $\begin{array}{l}\text { Capacidades } \\
\text { cuya demanda } \\
\text { crece }(\mathbf{2 0 2 2})\end{array}$ & $\begin{array}{l}\text { Capacidades cuya } \\
\text { demanda decrece } \\
(\mathbf{2 0 2 2})\end{array}$ \\
\hline $\begin{array}{l}\text { Pensamiento } \\
\text { analítico } \\
\text { innovación }\end{array}$ & $\begin{array}{l}\text { Pensamiento } \\
\text { analítico } \\
\text { innovación }\end{array}$ & $\begin{array}{l}\text { Destreza manual, } \\
\text { Resistencia } \\
\text { precisión }\end{array}$ \\
\hline $\begin{array}{l}\text { Resolución de } \\
\text { problemas } \\
\text { complejos }\end{array}$ & $\begin{array}{l}\text { Análisis } \\
\text { evaluación } \\
\text { sistemas }\end{array}$ & $\begin{array}{l}\text { Habilidades } \\
\text { visuales, auditivas } \\
\text { y del habla }\end{array}$ \\
\hline $\begin{array}{l}\text { Pensamiento } \\
\text { crítico y análisis }\end{array}$ & $\begin{array}{l}\text { Creatividad, } \\
\text { original } \\
\text { iniciativa }\end{array}$ & $\begin{array}{l}\text { Gestión de recursos } \\
\text { financieros, } \\
\text { materiales }\end{array}$ \\
\hline $\begin{array}{l}\text { Aprendizaje } \\
\text { activo } \\
\text { estrategias } \\
\text { aprendizaje }\end{array}$ & $\begin{array}{l}\text { Razonamiento, } \\
\text { resolución de } \\
\text { problemas e ideas }\end{array}$ & $\begin{array}{l}\text { Lectura, escritura, } \\
\text { matemáticas y } \\
\text { escucha activa }\end{array}$ \\
\hline $\begin{array}{l}\text { Creatividad, } \\
\text { original } \\
\text { iniciativa }\end{array}$ & $\begin{array}{l}\text { Pensamiento } \\
\text { crítico y análisis }\end{array}$ & $\begin{array}{lr}\text { Instalación } & \mathrm{y} \\
\text { mantenimiento } & \mathrm{de} \\
\text { tecnología } & \end{array}$ \\
\hline $\begin{array}{l}\text { Atención } \\
\text { detalle, } \\
\text { confiabilidad }\end{array}$ & $\begin{array}{l}\text { Diseño } \quad \text { y } \\
\text { programación de } \\
\text { tecnología }\end{array}$ & Gestión de personal \\
\hline $\begin{array}{l}\text { Inteligencia } \\
\text { emocional }\end{array}$ & $\begin{array}{l}\text { Liderazgo e } \\
\text { influencia social }\end{array}$ & $\begin{array}{l}\text { Control de calidad } \\
\text { y seguridad }\end{array}$ \\
\hline $\begin{array}{l}\text { Razonamiento, } \\
\text { resolución de } \\
\text { problemas } \\
\text { ideas }\end{array}$ & $\begin{array}{lr}\text { Aprendizaje } & \\
\text { activo } & \mathrm{y} \\
\text { estrategias } & \text { de } \\
\text { aprendizaje } & \end{array}$ & $\begin{array}{l}\text { Memoria, } \\
\text { habilidades } \\
\text { verbales, auditivas } \\
\text { y especiales }\end{array}$ \\
\hline $\begin{array}{l}\text { Liderazgo e } \\
\text { influencia social }\end{array}$ & $\begin{array}{l}\text { Inteligencia } \\
\text { emocional }\end{array}$ & $\begin{array}{l}\text { Coordinación y } \\
\text { gestión del tiempo }\end{array}$ \\
\hline 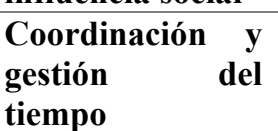 & $\begin{array}{l}\text { Resolución } \\
\text { problemas } \\
\text { complejos }\end{array}$ & $\begin{array}{l}\text { Uso de la } \\
\text { tecnología, } \\
\text { monitoreo y control }\end{array}$ \\
\hline
\end{tabular}

Nota. World Economic Forum $\left(2018^{\mathrm{a}}\right)$, tomado de (The Future of Jobs Report 2018, p.12).

El cuanto al desarrollo de talentos que se requieren tenemos a la orientación al cliente, trabajo en equipo, a la capacidad de comunicación, facultad de ejecución y la negociación, estas son algunos requerimientos que se solicitan actualmente siendo la brecha muy reducida que se demandan para el 2023, se considera importante el trato al cliente el brindar un buen servicio trabajando en equipo y el manejo de comunicación como se muestra en el grafico 1. 


\section{Gráfico 1}

El futuro del empleo y las competencias profesionales del futuro:
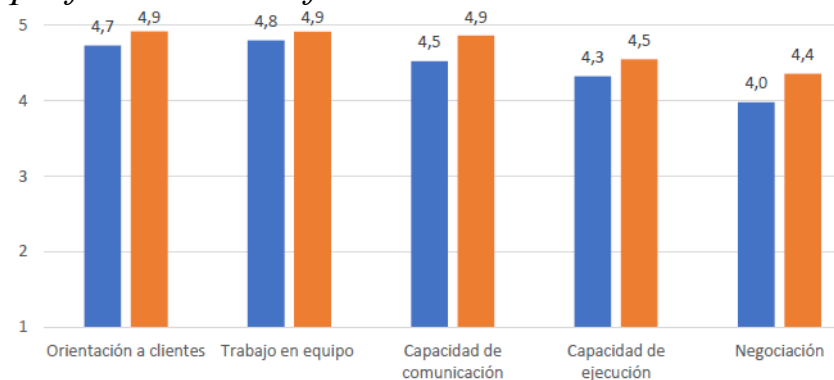

- $2018=2023$

Nota. Tomado de fuente: la perspectiva de las empresas (2020).

\section{Gráfico 2}

El futuro del empleo y las competencias profesionales del futuro:

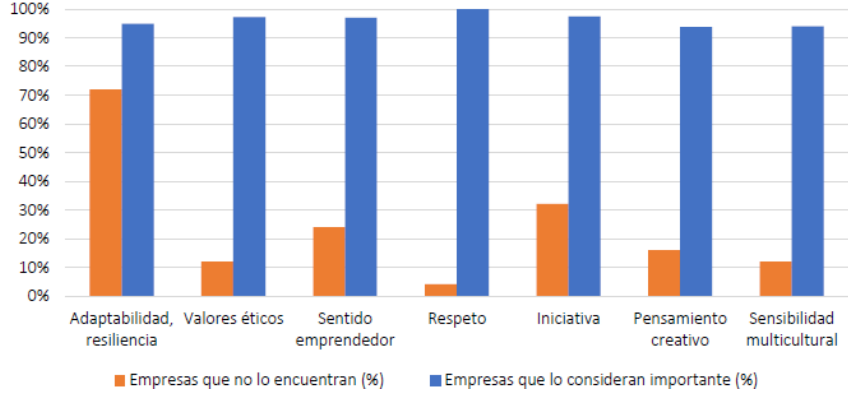

Nota. la perspectiva de las empresas (2020).

En la gráfica 2, se puede observar que existen competencias que no encuentran las empresas en los egresados de las diferentes profesiones, es preocupante que se resalte el respecto que no llega ni al $5 \%$, como un $12 \%$ se tienen los valores éticos, el $15 \%$ el pensamiento creativo y con un $11 \%$ de falta de sensibilidad multicultural, por tal motivo se tomaron en cuenta la propuesta del plan de estudios cubrir algunas de las necesidades que requieren.

Ante la necesidad de impulsar diferentes competencias y habilidades, que ayuden a los estudiantes a realizar sus actividades laborales lo mejor posible, se procedió a ser una evaluación sobre lo que ofrece el plan de estudios de la carrera de Ingeniería en Alimentos actual. Derivado de los resultados del estudio se desarrolló una propuesta para la asignatura de Estadística, que a continuación se presenta en la tabla siguiente.

Al manejar disciplinas interdisciplinares como programación, microbiología, métodos cuantitativos, ingeniería industrial, envases, embalaje, diseño de experimentos, y ser integradas al área de la estadística se ha logrado aplicar contenidos esenciales de cada una de ellas al trabajarlas en conjunto con datos recopilados de un problema planteado por los grupos de trabajo se desarrollan diferentes competencias laborales.

Tomando en consideración las necesidades que se demandan se realizó un comparativo del programa actual contra un procedimiento modificado que justifica la propuesta expuesta en el cuadro1, para el rediseño del plan de estudios de alumnos de la carrera de ingeniería en Alimentos, se incluyeron el desarrollo de diferentes capacidades necesarias en la vinculación con otras asignaturas, que los lleven a tener éxito en su trabajo profesional, ver cuadro 2 .

\section{Cuadro 2}

Cuadro comparativo de los programas

\section{PROGRAMA \\ PROGRAMA MODIFICADO} ACTUAL

Tiempo 3 horas por Tiempo 6 horas por semana semana

Sesiones: 2 de 1.5 h
$\begin{aligned} & \text { Programas } \\ & \text { Académicos: Ing. en }\end{aligned}$


probabilidad, distribuciones probabilidad, pruebas de hipótesis, métodos de inferencia estadística, regresión lineal, análisis de varianza, intervalos de confianza.

\begin{tabular}{lrll} 
Competencias & y & Competencias y Habilidades: \\
Habilidades: solución & pensamiento crítico, manejo de \\
de problemas, & cantidades grandes & de \\
pensamiento crítico, & información, resolución de \\
trabajo en equipo, & problemas, trabajo (colaborativo y \\
manejo de datos, & en equipo), manejo de grupos de \\
comunicación. & & trabajo, comunicación, resiliencia \\
& & y manejo de emociones. \\
\hline
\end{tabular}

Nota. Rediseño del plan de estudios, elaboración propia (2020).

Al tomar los elementos necesarios para vincular las áreas que se desea trabajar, se debe conocer el tiempo que se pasa en el aula y en la unidad académica, en conseguir integrar nuevas competencias y habilidades, los estudiantes al abordar problemas emplean la curiosidad al realizar una experimentación, se estimula su creatividad y la originalidad (Centro de Rediseño Curricular y OECD, 2012).

\section{Análisis}

El estudio se implementó a 80 estudiantes de segundo semestre, que cursan la carrera de ingeniería en Alimentos de la Unidad Profesional Interdisciplinaria de Biotecnología durante el segundo periodo 2018, que cursaron la asignatura de Estadística, se realizaron cambios en las formas de plantear los contenidos al vincularlos con otras áreas de su profesión, encontramos los siguiente.

Se muestra a continuación las derivaciones que se obtuvieron en cada curso de Estadística, primero se analizó la clase sin modificaciones al programa de estudios, donde el realiza una exposición magistral ante los alumnos y los estudiantes toman una actitud pasiva receptora de información, sin tener una idea clara de cómo poder vincularlos temas vistos con otras materias los resultados se muestran en los siguientes gráficos.

\section{Gráfico 3}

Promedio de evaluaciones del curso normal de estudiantes de ingeniería en Alimentos de la unidad profesional interdisciplinaria de biotecnología.

CURSO DE ESTADISTICA NORMAL

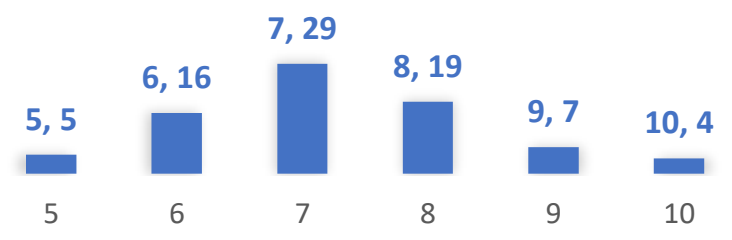

Nota. Promedio de evaluación, elaboración propia (2020).

\section{Gráfico 4}

Competencias y habilidades de un curso normal de estadistica en la unidad Profesional interdisciplinaria de biotecnología.

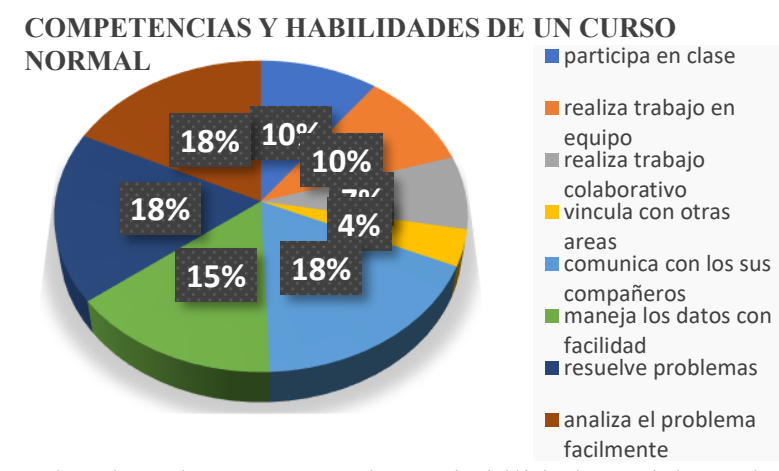

Nota. Evaluación de competencias y habilidades, elaboración propia (2020).

Se examina en el gráfico 1, que los promedios de los estudiantes se encuentran entre 6-8, de acuerdo con sus evaluaciones finales del curso, en la gráfica 2 , se observa la vinculación con otras áreas es casi nula con $4 \%$, el trabajo colaborativo un $7 \%$, la participación en clase y la tarea en equipo es de un $10 \%$, el manejo de datos aplicando un software es del $15 \%$, en el análisis del problema y la realización del ejercicio $18 \%$.

Los cambios que se realizaron al programa de estudios de Estadística consistieron, en dar una nueva visión a los contenidos descriptos en el curso, mostrando la relación que se tiene con diferentes asignaturas que cursan en ese momento y la aplicación de estas con otras materias, se percataron de la utilidad de enlazar algunas disciplinas, les pareció practico y útil como se muestra en la siguiente grafica. 


\section{Gráfico 5}

Promedio de evaluaciones al modificar el programa de estudios de estudiantes de ingeniería en Alimentos de la unidad profesional interdisciplinaria de biotecnología.

CURSO DE ESTADISTICA MODIFICADO

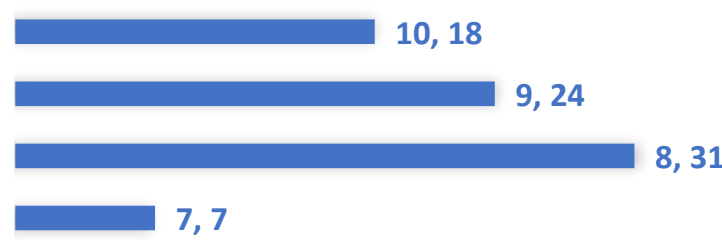

Nota. Cursos de estadísticas modificado, elaboración propia (2020).

\section{Gráfico 6}

Competencias y habilidades del curso modificado de estadística en la unidad Profesional interdisciplinaria de biotecnología.

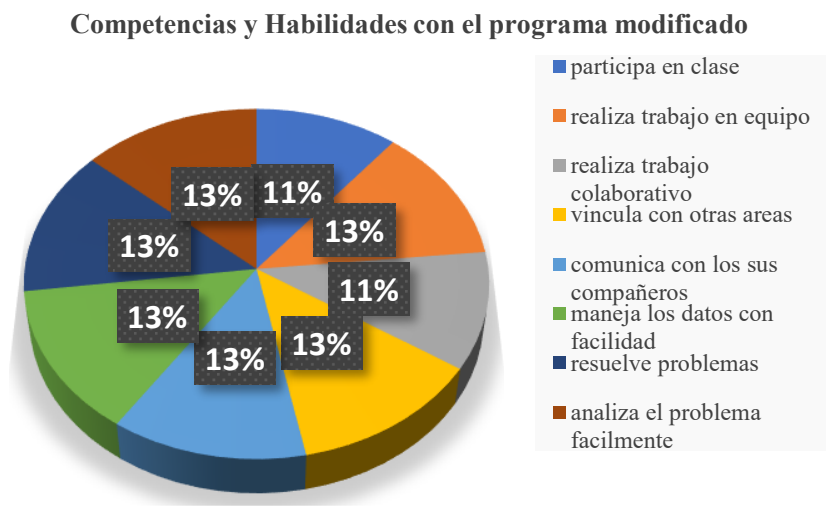

Nota. Competencias y habilidades en el programa modificado, elaboración propia (2020).

Al realizar los cambios del enfoque de los contenidos del programa de estudios, se muestra en el gráfico 3. Que los promedios finales están entre 8-9, y en la gráfica 4 . Se observa que la participación en clase y el trabajo colaborativo tienen el 11\%, se logró un aumento en la tarea en equipo, se alcanzó vincularan con otras materias, mejoro la comunicación con sus compañeros teniendo un $13 \%$.

\section{Resultados}

Se observa una diferencia significativa entre la impartición de los contenidos de la asignatura en forma tradicional, contra un nuevo modo abordar los temas en manera práctica vinculando con materias que trabajan en los diferentes laboratorios. Se nota un incremento significativo en las evaluaciones de los alumnos, y el desarrollo de las diversas competencias y habilidades al encontrar como aplicar la estadística.

Derivado del análisis presentado anteriormente, se observa que los cambios realizados en los programas de estudios son cruciales para los estudiantes de la carrera de ingeniería en Alimentos, en adquirir competencias que les permitirán lograr resolver problemas en plantas de transformación químico-biológicas, en la creación del diseño de bioprocesos industriales, en emitir una valorización de los diseños creados.

Como en la participación del esbozo de la instalación de procesadoras biotecnológicas, organizando la coordinación del aseguramiento de calidad, mejorando los métodos químicos $\mathrm{y}$ biotecnológicos, incluyendo puntos críticos de control, conociendo la legislación vigente en su área de incidencia, procurando tener un impacto positivo socioeconómico y ambiental, al aplicar técnicas de administración alcanzando objetivos de operación y bosquejos de proyectos tecnológicos.

Con la importancia de contar con la capacidad de coordinar equipos de trabajo, en el manejo y aplicación de nuevas tecnologías de la comunicación, en la dirección de grupos de colaboración, y el desarrollo de procesos creativos, impulsando los proyectos de elaboración en la manera de procesar diferentes elementos, con una creatividad en los métodos de conservación, diseñando diversas metodologías que aumenten la productividad.

\section{Conclusiones}

Ante la necesidad de formar a estudiantes de ingeniería en Alimentos en diversas competencias y habilidades requeridas por el sector industrial, y no contar con una vinculación permanente entre la escuela y la industria, hace que resulte difícil desarrollar estas necesidades, por lo que realizamos algunos estudios con las diferentes asignaturas que se encuentran en la unidad profesional interdisciplinaria de biotecnología.

Al término de este estudio se ha concluyo que fueron provechosos los resultados obtenidos durante el ejerció, no se logró alcanzar un porcentaje alto de estudiantes que vincularan los contenidos de la asignatura, con las otras materias que llevaban al 
mismo tiempo, externaron que en un principio no tenían claro cómo aplicar los temas, en el transcurso el semestre se fue estructurando la manera de utilizar lo visto en clase.

Cuando se toma la decisión de realizar cambios a los programas de estudio, sugerimos que se tome a un grupo como piloto de prueba, se apliquen los contenidos que se planean colocar en el programa de estudios, y se vayan realizando un monitoreo constante de los temas que se proponen a desarrollar son adecuados, y van de acuerdo con los tiempos planeados por la estructura curricular.

Es fácil el monitorear los avances que se tienen y las dificultades que se presentan poder corregirlas rápidamente, con estos cambios que se proponen se espera fomentar su creatividad e innovación, en la solución de toma de decisiones, como las maneras de obtener resultados rápidos $\mathrm{y}$ confiables, ante este nuevo paradigma pedagógico, los procesos de enseñanza-aprendizaje.

Hacer capaces a los estudiantes de manejar hoy en día información a gran escala, el sistema educativo requiere transformarse, así ofrecer aprendizajes sostenibles, auto suficientes, autodidactas, que los encaminen a un aprendizaje complejo, creando talentos que cubran diferentes necesidades del sector industrial con capacidad de poder lograr grandes cosas en su quehacer profesional.

Los docentes y los sistemas educativos deben de replantearse, en crear nuevos espacios formativos, al enfrentase a varios retos con diferentes escenarios, partiendo de metodologías, adecuadas a los procesos de enseñanza-aprendizaje, así garantizar una formación de calidad a los futuros profesionales, en incorporarse a una nueva industria. Para esto es necesario restructurar cambios en la educación.

Tomando en cuenta las diversas capacidades que hoy en día requieren de personas que desarrollen y manejen tecnologías, aplicando ciertas habilidades, esto no es algo nuevo, todos los días en las aulas tenemos la oportunidad de fortalecer estos talentos, por ello es relevante involucrar al docente a que sean desarrollado en los estudiantes el interés por crear, diseñar nuevas formas de encontrar respuestas.

Concluimos, que la propuesta logró el objetivo planteado al vincular varias materias de primer semestre, se espera que se incorporen estos cambios ya establecidos a la planeación curricular y corregir los errores que se encontraron, seguir innovando nuevas metodologías de enseñanza aprendizaje, dando sentido a las asignaturas que se imparten en la carrera de ingeniería en Alimentos.

\section{Referencias}

Arribas-Urrutia, O Islas-Carmona, F Gutiérrez Cortés (2019): De prosumidores a observadores: una tendencia emergente en Internet y en los jóvenes ecuatorianos. Resultados del estudio del World Internet Project, Ecuador. Revista Latina de Comunicación Social, 74, pp. 969 a 996. http://www.revistalatinacs.org/074paper/1367/50es.html DOI: 10.4185/RLCS-2019-1367

Barráez, D. P. (2020). La educación a distancia en los procesos educativos: Contribuye significativamente al aprendizaje. Revista Tecnológica-Educativa Docentes 2.0, 8(1), 41-49. Recuperado a partir de https://ojs.docentes20.com/index.php/revistadocentes $20 /$ article/view/91

Barreto, R. \& Diaz-Granados, F. (2017). Las TIC en la educación superior. Experiencias de Innovación. Universidad del Norte. Colombia.

Bernal-Villamarin, S. C. (2015). Diseño y creación de contenidos educativos digitales a través de herramientas web 2.0. Revista Nuevas ideas en Informática Educativa. TISE.

Carvajal, J. H. (2017). La cuarta Revolución Industrial o Industria 4.0 y su impacto en la educación superior en ingeniería en Latinoamérica y el Caribe. LACCEI, pp. 1- 5.

Contreras-Maradey, F. D. (2017). Apropiación tecnológica para la incorporación efectiva de recursos educativos abiertos. Revista Apertura. Vol. 9, Núm. 1. Universidad de Guadalajara.

Gértrudix-Barrio, M., Álvarez García, S., Galisteo, A., Del Valle, M. C., Gálvez de la Cuesta, F. (2007) Acciones de diseño y desarrollo de objetos educativos digitales: programas institucionales. Artículo. Revista de Universidad y Sociedad del Conocimiento.

Hernández, L. (2020). Liderazgo sostenible como estrategia de cambio en el sistema educativo venezolano. Revista TecnológicaEducativa Docentes 2.0,8(1), 26-32. Recuperado de: https://ojs.docentes20.com/index.php/revistadocentes20/article/view/85

López-Jiménez, D. (2009). La naturaleza de las tecnologías de información y comunicación: las TIC como determinantes de la organización y de la sociedad de la información. Universidad de La Sabana. Recuperado de: http://dspace.uhemisferios.edu.ec:8080/xmlui/handle/1234567 $89 / 453$

Mayorga, M. (2020). Conocimiento, aplicación e integración de las TIC - TAC y TEP por los docentes universitarios de la ciudad de Ambato. Revista Tecnológica-Educativa Docentes 2.0, 9(1), 511. https://doi.org/10.37843/rted.v9i1.101.

Mujica-Sequera, R. (2020). E-Learning como estrategia pedagógica en la educación superior. Revista Tecnológica-Educativa Docentes 2.0, 9(1), 37-41. https://doi.org/10.37843/rted.v9i1.103 
Naji, M. J. (2018). Industria 4.0, competencia digital. Revista Internacional y Comparada de Relaciones Laborales y Derecho al Empleo, pp. 164-194

Oppenheimer, A. (2018) ¡Sálvese quien pueda! El futuro del trabajo en la era de la automatización. Capítulo 7. Ciudad de México

Panza de Ferrer, N. (2020). Docente universitario, alfarero de vidas.... Revista Tecnológica-Educativa Docentes 2.0, 8(1), 60-65. Recuperado de: https://ojs.docentes20.com/index.php/revistadocentes20/article/view/106

Resa López, M. (2020). Tecnología y Enseñanza Situada. Revista Tecnológica-Educativa Docentes 2.0, 8(1), 33-40. Recuperado a partir de https://ojs.docentes20.com/index.php/revistadocentes $20 /$ article/view/83

Sánchez, L. (2020). Impacto del Aula Virtual en el Proceso de Aprendizaje de los Estudiantes de Bachillerato General. Revista Tecnológica-Educativa Docentes 2.0,9(1), 75-82. https://doi.org/10.37843/rted.v9i1.105

NEA. (2010). Preparing 21 st Century Students for a Global Society:An Educator's Guide to 'The Four Cs'. Washington D.C.: National Education Association (NEA). https://nea.org/tools/52217.htm

P21. (2007a). The Intellectual and Policy Foundations of the 21st Century Skills Framework. Washington D.C.: Partnership for 21st Century Skills. Recuperado de: https://route21.p21.org/images/stories/epapers/skills_foundati ons_final.pdf

P21. (2007b. 21st Century Curriculum and Instruction. Washington D.C.: Partnership for 21st Century Skills. Recuperado de: https://route21.p21.org/images/stories/epapers/r21_ci_epaper. pdf

P21. (2008). 21st Century Skills, Education \& Competitiveness.Washington D.C.: Partnership for 21st Century Skills. Recuperado de: https://www.p21.org/storage/documents/21st_century_skills_e ducation_and_competitiv eness_guide.pdf

P21. (2011). Framework for 21 st Century Learning. Washington D.C.: Partnership for 21 st Century Skills. Recuperado de: https://www.p21.org/storage/documents/1._p21_framework_ 2-pager.pdf

P21. (2013). Reimagining Citizenship for the 21st Century: A Callto Action for Policymakers and Educators. Washington D.C.: Partnership for 21st Century Skills. Recuperado de: https://www.p21.org/storage/documents/Reimagining_Citizen ship_21st_Century_webversion.pdf

Prensky, M. (2012). From Digital Natives to Digital Wisdom: Hopeful Essays for 21st Century Learning. Thousand Oaks, California: Corwin.

Punie, Y. (2007). Learning spaces: an ICT-enabled model of future learning in the knowledge-based society. European Journal of Education. Vol. 42, No. 2, pág. 185-199. Recuperado de: http://onlinelibrary.
UNESCO. (2013d). Informe de Seguimiento de la EPT en el Mundo, 2013-2014. París: UNESCO. Recuperado de: http://unesdoc.unesco.org/images/0022/002256/225654s.pdf

UNESCO y UNICEF. (2013). Envisioning Education in the Post-2015 Development Agenda: Executive Summary. París: UNICEF y UNESCO. Recuperado http://en.unesco.org/post2015/sites/post2015/files/Post2015 en web. 